\title{
TAMENESS AND GEODESIC CORES OF SUBGROUPS
}

\author{
RITA GITIK \\ (Received 13 December 1999; revised 15 February 2000) \\ Communicated by C. F. Miller
}

\begin{abstract}
Let $N$ be a finitely generated normal subgroup of a finitely generated group $G$. We show that if the trivial subgroup is tame in the factor group $G / N$, then $N$ is tame in $G$. We also give a short new proof of the fact that quasiconvex subgroups of negatively curved groups are tame. The proof utilizes the concept of the geodesic core of the subgroup and is related to the Dehn algorithm.
\end{abstract}

2000 Mathematics subject classification: primary 57M07, 57M30, 57N10, 20F32, $20 \mathrm{~F} 34$.

\section{Introduction}

A 3-manifold $M$ is called a missing boundary manifold if it can be embedded in a compact manifold $\bar{M}$ such that $\bar{M} \backslash M$ is a closed subset of the boundary of $\bar{M}$.

One of the long-standing open problems in the field of 3-manifolds is the missing boundary manifold conjecture due to Simon ([Sim]). He conjectured that if $M_{0}$ is a compact orientable irreducible 3-manifold, and $M$ is the cover of $M_{0}$ corresponding to a finitely generated subgroup of $\pi_{1}\left(M_{0}\right)$, then $M$ is a missing boundary manifold.

This conjecture has been verified in many special cases, (see [Kir, page 151] and [Gab] for additional information), however the general case is still open. In the special case when $M$ has no boundary and $\pi_{1}(M)$ is finitely generated, $M$ is a missing boundary manifold if and only if it is homeomorphic to the interior of a compact manifold. In this case $M$ has finitely many ends, so it is a missing boundary manifold if and only if each of its ends is tame. (An end is tame if it is homeomorphic to a product (closed surface) $\times[0, \infty)$.) Hence the missing boundary manifold conjecture is also known as the tame ends conjecture.

Thurston showed in [Thu] that if $M_{0}$ is hyperbolic, geometrically finite and has infinite volume, then every cover of $M_{0}$ with a finitely generated fundamental group

(c) 2000 Australian Mathematical Society $0263-6115 / 2000 \$ A 2.00+0.00$ 
has tame ends. Bonahon showed in [Bon] that any hyperbolic manifold $M$ with finitely generated $\pi_{1}(M)$ has tame ends, provided $\pi_{1}(M)$ is not a free product.

Tucker proved in [Tuc] that a non-compact orientable irreducible 3-manifold $M$ is a missing boundary manifold if and only if for any compact submanifold $C$ of $M$ the group $\pi_{1}(M \backslash C)$ is finitely generated.

This observation made it possible to reduce the missing boundary manifold conjecture to a group-theoretic problem. The first results in this direction were obtained by Casson and Poénaru, (see [Poé]). Mihalik introduced the notion of a tame pair of groups in [Mi1]. Let $M_{0}$ be a 3-manifold with the fundamental group $G$, and let $M$ be a cover of $M_{0}$ corresponding to a subgroup $H$ of $G$. Then the pair $(G, H)$ is tame if and only if $M$ is a missing boundary manifold. This approach resulted in various group-theoretical results which implied some special cases of the tame ends conjecture, (see [Mi1, Mi2]).

However, the concept of a tame subgroup seems to be of independent interest. For example, it is not known weather the trivial subgroup is tame in any finitely generated group. Also there are no examples of a non-tame pair $(G, H)$ with both $H$ and $G$ finitely generated.

In this paper we introduce a different, though equivalent, definition of a tame subgroup. Let $M_{0}$ be a compact orientable irreducible 3-manifold, let $G=\pi_{1}\left(M_{0}\right)$, and let $H$ be a subgroup of $G$. Choose the presentation $G=\langle X \mid R\rangle$. Let $K$ be the standard 2-complex representing $G$, that is, $K$ has one vertex, $K$ has an edge for any generator $x \in X$, and $K$ has a 2-cell for any relator $r \in R$. Let Cayley ${ }_{2}(G)$ be the universal cover of $K$, and let Cayley $_{2}(G, H)$ be the cover of $K$ corresponding to a subgroup $H$ of $G$.

Let $\tilde{M}_{0}$ be the universal cover of $M_{0}$ and let $M$ be the cover of $M_{0}$ corresponding to $H$. Then Cayley ${ }_{2}(G)$ imbeds quasi-isometrically in $\tilde{M}_{0}$, and $\operatorname{Cayley}_{2}(G, H)$ embedded quasi-isometrically in $M$. Let $C$ be a compact submanifold of $M$. It is easy to see that the fundamental group of $\operatorname{Cayley}_{2}(G, H) \backslash C$ is finitely generated if and only if the fundamental group of $M \backslash C$ is. So Tucker's theorem implies that $M$ is a missing boundary manifold if and only if $\pi_{1}\left(\operatorname{Cayley}_{2}(G, H) \backslash C\right)$ is finitely generated for any finite subcomplex $C$ of Cayley $_{2}(G, H)$.

This discussion motivates the following definition.

DEFINITION 1. A subgroup $H$ of a group $G$ is tame in $G$ if for any finite subcomplex $C$ of Cayley $2(G, H)$ the group $\pi_{1}\left(\right.$ Cayley $\left._{2}(G, H) \backslash C\right)$ is finitely generated.

\section{Preliminaries}

Let $M_{0}$ be a compact orientable irreducible 3-manifold, and let $G=\pi_{1}\left(M_{0}\right)$. Choose a presentation $G=\langle X \mid R\rangle$. Let $X^{*}=\left\{x, x^{-1} \mid x \in X\right\}$, and for $x \in X$ define 
$\left(x^{-1}\right)^{-1}=x$. Recall that the Cayley graph of $G$, denoted Cayley $(G)$, is an oriented graph whose set of vertices is $G$ and the set of edges is $G \times X^{*}$, such that an edge $(g, x)$ begins at the vertex $g$ and ends at the vertex $g x$.

DEFINITION 2. Let $H$ be a subgroup of $G$, and let $\{H g\}$ be the set of right cosets of $H$ in $G$. The relative Cayley graph of $G$ with respect to $H$ (or the coset graph) is an oriented graph whose vertices are the cosets $\{\mathrm{Hg}\}$, the set of edges is $\{\mathrm{Hg}\} \times \mathrm{X}^{*}$, such that an edge $(H g, x)$ begins at the vertex $H g$ and ends at the vertex $H g x$. We denote it Cayley $(G, H)$.

A word in $X$ is any finite sequence of elements of $X^{*}$. Denote the set of all words in $X$ by $W(X)$, and denote the equality of two words by $\equiv$.

DEFINITION 3. The label of a path

$$
p=\left(H g_{1}, x_{1}\right)\left(H g_{1} x_{1}, x_{2}\right) \cdots\left(H g_{1} x_{1} \cdots x_{n-1}, x_{n}\right) \text { in Cayley }(G, H)
$$

is the function $\operatorname{Lab}(p) \equiv x_{1} x_{2} \ldots x_{n} \in W(X)$.

As usual, we identify the word $\operatorname{Lab}(p)$ with the corresponding element in $G$.

Note that Cayley $(G)$ is the 1-skeleton of $\operatorname{Cayley}_{2}(G)$, and Cayley $(G, H)$ is the 1-skeleton of Cayley $_{2}(G, H)$. The following example illustrate the definitions.

LEMMA 1. Let $H$ be a subgroup of a group $G$, and let $H_{0}$ be a finite index subgroup of $H$. Then $H$ is tame in $G$ if and only if $H_{0}$ is.

Proof. Let $\phi:$ Cayley $\left(G, H_{0}\right) \rightarrow$ Cayley $(G, H)$ be the covering map, and let $C$ be a finite subcomplex of Cayley $(G, H)$. As $H_{0}$ is a finite index subgroup of $H, \phi$ is a finite to one map. Then $\phi^{-1}(C)$ is a finite subcomplex of Cayley $\left(G, H_{0}\right)$. If $H_{0}$ is tame in $G$, then $\pi_{1}$ (Cayley $\left.\left(G, H_{0}\right) \backslash \phi^{-1}(C)\right)$ is finitely generated. As $\phi$ is a finite covering map from Cayley $\left(G, H_{0}\right) \backslash \phi^{-1}(C)$ to Cayley $(G, H) \backslash C$, it follows that $\pi_{1}$ (Cayley $\left.\left(G, H_{0}\right) \backslash \phi^{-1}(C)\right)$ is a finite index subgroup of $\pi_{1}(\operatorname{Cayley}(G, H) \backslash$ $C$ ), hence $\pi_{1}$ (Cayley $(G, H) \backslash C$ ) is finitely generated, so $H$ is tame in $G$. In the other direction, if $C_{0}$ is a finite subcomplex in Cayley $\left(G, H_{0}\right)$, then $C=\phi\left(C_{0}\right)$ is a finite subcomplex in Cayley $(G, H)$. If $H$ is tame in $G$, then $\pi_{1}(\operatorname{Cayley}(G, H) \backslash C)$ is finitely generated, hence $\pi_{1}$ (Cayley $\left.\left(G, H_{0}\right) \backslash \phi^{-1}(C)\right)$ is finitely generated. But Cayley $\left(G, H_{0}\right) \backslash C_{0}$ is constructed from Cayley $\left(G, H_{0}\right) \backslash \phi^{-1}(C)$ by adding a finite complex, hence $\pi_{1}$ (Cayley $\left.\left(G, H_{0}\right) \backslash C_{0}\right)$ is finitely generated, so $H_{0}$ is tame in $G$. 


\section{Normal subgroups}

Let $Y$ be a subset of a generating set of a group $G$, and let $p$ be a path in $\operatorname{Cayley}(G, H)$. We say that $p$ is labeled with $Y$ if $\operatorname{Lab}(p)$ is a word in $Y^{*}$. We denote the inverse of a path $p$ by $\bar{p}$.

THEOREM 1. Let $N$ be a finitely generated normal subgroup of a finitely generated group $G$. Then $N$ is tame in $G$ if the trivial subgroup is tame in the factor group $G / N$.

PROOF. Let $\rho: G \rightarrow G / N$ be the projection map. Let $X=\left\{x_{1}, \ldots, x_{m}\right\}$ be a generating set for $N$ and let $Y=\left\{y_{1}, \ldots, y_{k}\right\}$ be a subset of $G$ such that $\left\{\rho\left(y_{1}\right), \ldots, \rho\left(y_{k}\right)\right\}$ is a generating set for $G / N$. Choose the presentation $\langle X, Y \mid R, T\rangle$ for $G$, where $R$ consists of the conjugation relators of the form $\left\{y_{i} x_{j} y_{i}^{-1} n_{j} \mid n_{j} \in\right.$ $N, 1 \leq i \leq k, 1 \leq j \leq m\}$. In this presentation Cayley $(G, N)$ is isomorphic to a product of Cayley $(G / N)$ and a wedge of $m$ circles, one circle for each generator of $N$, however Cayley $_{2}(G, N)$ need not be a product. Let $C$ be a finite subcomplex in Cayley $_{2}(G, N)$. Choose a basepoint $v$ in Cayley $(G, N) \backslash C$ and let $l$ be a loop in Cayley $(G, N) \backslash C$ beginning at $v$. Then $l$ can be written as a product $l_{1} l_{2} \cdots l_{n}$, where each $l_{i}$ is a loop beginning at $v$, all the loops $l_{2 i}$ are labeled with $Y$, and all the loops $l_{2 i-1}$ have the form $p_{2 i-1} q_{2 i-1} \bar{p}_{2 i-1}$, where $p_{2 i-1}$ is labeled with $Y$ and $q_{2 i-1}$ is labeled with $X$.

We claim that using the conjugation relators from $R$ each loop $l_{2 i-1}$ is homotopic in $\operatorname{Cayley}_{2}(G, N) \backslash C$ to a loop $t_{2 i-1}$ which begins at $v$ and is labeled with $Y$. The proof is by induction on the length of the path $p_{2 i-1}$. If its length is 0 , then we can take $l_{2 i-1}=t_{2 i-1}$. Assume that the statement is true if $p_{2 i-1}$ is shorter than $n$. Consider a loop $l_{2 i-1}$ with $p_{2 i-1}$ of length $n$, so $p_{2 i-1}=e_{1} \cdots e_{n}$ and $q=e_{1}^{\prime} \cdots e_{l}^{\prime}$, where $e_{i}$ and $e_{j}^{\prime}$ are edges in Cayley $(G, N) \backslash C$. Consider a path $e_{n} e_{1}^{\prime} \bar{e}_{n}$. As $\operatorname{Lab}\left(e_{n}\right) \operatorname{Lab}\left(e_{1}^{\prime}\right) \operatorname{Lab}\left(e_{n}\right)^{-1}$ is a part of a conjugation relator from $R$, there exists a path $s_{1}$ in $\operatorname{Cayley}(G, H)$ labeled with $X$ such that $e_{n} e_{1}^{\prime} \bar{e}_{n} s_{1}$ bounds a 2-cell in Cayley $(G, H)$. This cell provides a homotopy of $e_{n} e_{1}^{\prime} \bar{e}_{n}$ to $s_{1}$. Similarly, for $1 \leq j \leq l$ we construct a homotopy of $e_{n} e_{j}^{\prime} \bar{e}_{n}$ to a path $s_{j}$ labeled with $X$. Combining these homotopies we obtain a homotopy of $l_{2 i-1}$ to a path $e_{1} \cdots e_{n-1} s_{1} \cdots s_{l} \bar{e}_{n-1} \cdots \bar{e}_{1}$, and we can use the inductive assumption. It remains to show that the paths $s_{j}$ and the 2-cell bounded by $e_{n} e_{j}^{\prime} \bar{e}_{n} s_{j}$ belong to Cayley $(G, H) \backslash C$. Indeed, by construction, the path $e_{n} e_{j}^{\prime} \bar{e}_{n}$ is in Cayley $(G, H) \backslash C$. As $C$ is a closed complex, it follows that the 2-cell bounded by $e_{n} e_{j}^{\prime} \bar{e}_{n} s_{j}$ lies in Cayley $(G, H) \backslash C$. Also all the edges in $s_{j}$ have the same common initial and terminal vertex, which belongs to the path $l_{2 i-1}$ and lies in the compliment of $C$. Hence all the edges of $s_{j}$ lie in the compliment of $C$, proving the statement. Therefore $l$ is homotopic to a product $t_{1} l_{2} t_{3} \cdots l_{n}$, where each $l_{2 i}$ is a loop beginning at the basepoint $v$, labeled with $Y$, and all the loops $t_{2 i-1}$ are labeled with $X$. By construction, all 
the vertices of the paths $t_{2 i-1}$ coincide with the basepoint $v$, so all $t_{2 i-1}$ belong to the 0 -neighbourhood of $v$. However, if the trivial subgroup is tame in $G / N$, then the paths $l_{2 i}$ can be homotoped to a bounded neighborhood of $v$ in Cayley $(G / H) \backslash C$, so they can be homotoped to a bounded neighbourhood of $v$ in Cayley $(G, H) \backslash C$. Therefore, if the trivial subgroup is tame in $G / N$ any loop $l$ in Cayley $(G, N) \backslash C$ can be homotoped to a loop in a bounded neighborhood of $v$, so $N$ is tame in $G$.

Recall that Mihalik proved in [Mi2] that if $N$ is a normal subgroup of $G$ and the groups $G, N$ and $G / N$ are finitely presented, then any finitely generated subgroup of infinite index in $N$ is tame in $G$. Lemma 1 and Theorem 1 show that if the trivial subgroup is tame in $G / N$, then any finite index subgroup of $N$ is tame in $G$.

\section{Geodesic core of a subgroup}

A geodesic in the Cayley $(G)$ is a shortest path joining two vertices. Let $\rho_{H}$ : $\operatorname{Cayley}(G) \rightarrow$ Cayley $(G, H)$ be the projection map: $\rho_{H}(g)=H g$ and $\rho_{H}(g, x)=$ $(H g, x)$.

Definition 4. (See [Git].) A geodesic in $\operatorname{Cayley}(G, H)$ is the image of a geodesic in Cayley $(G)$ under the projection $\rho_{H}$. The geodesic core of Cayley $(G, H)$ is the subgraph of Cayley $(G, H)$ which consists of the union of all the vertices and all the edges which belong to closed geodesics in Cayley $(G, H)$ beginning at the vertex $H \cdot 1$. We denote it $\operatorname{Core}(G, H)$.

Note that any path $p$ in Cayley $(G, H)$ which begins at $H \cdot 1$ ends at $H \cdot \operatorname{Lab}(p)$, so a path $p$ beginning at $H \cdot 1$ is closed, if and only if $\operatorname{Lab}(p) \in H$.

A subgroup $H$ of $G$ is $K$-quasiconvex in $G$ if any geodesic in the Cayley graph of $G$ with the endpoints in $H$ belongs to the $K$-neighbourhood of $H$. A subgroup is quasiconvex in $G$ if it is $K$-quasiconvex in $G$ for some $K$.

The following lemma from [Git] gives an important equivalent definition of quasiconvexity.

LEMMA 2. A subgroup $H$ of a group $G$ is $K$-quasiconvex if and only if $\operatorname{Core}(G, H)$ belongs to the $K$-neighbourhood of $H \cdot 1$ in $\operatorname{Cayley}(G, H)$.

PROOF. Let $\gamma$ be a closed geodesic in $\operatorname{Core}(G, H)$ beginning at $H \cdot 1$. Then $\operatorname{Lab}(\gamma) \in H$ and $\gamma$ is the image of a geodesic $\tilde{\gamma}$ in Cayley $(G)$ which begins at 1 with $\operatorname{Lab}(\tilde{\gamma}) \equiv \operatorname{Lab}(\gamma)$ under the projection map. But the projection map preserves distances from $H$, and it maps $H \subset \operatorname{Cayley}(G)$ onto $H \cdot 1 \in \operatorname{Cayley}(G, H)$, so $\gamma \subset N_{K}(H \cdot 1) \subset$ Cayley $(G, H)$ if and only if $\tilde{\gamma} \subset N_{K}(H) \subset \operatorname{Cayley}(G)$. 
The following example illustrate the definitions.

EXAMPLE 1. Let $G=\left\langle x, y \mid x y x^{-1} y^{-1}\right\rangle$ be the standard presentation of $Z \times Z$, and let $H=\langle x\rangle$ be a subgroup of $G$. For any vertex $x^{n}$ of $H$ there exists a unique geodesic in Cayley $(G)$ connecting 1 and $x^{n}$, namely the horizontal path $p$ with $\operatorname{Lab}(p) \equiv x^{n}$ so $H$ is a 0-quasiconvex subgroup of Cayley $(H)$. Cayley $(G, H)$ can be described as follows: the set $\left\{H y^{n}, n \in Z\right\}$ is the set of all cosets of $H$ in $G$, hence we consider it as the set of vertices of Cayley $(G, H)$. The edge $\left(H y^{n}, y\right)$ begins at the vertex $H y^{n}$ and ends at the vertex $H y^{n} y=H y^{n+1}$. The edge $\left(H y^{n}, x\right)$ begins at the vertex $H y^{n}$ and ends at the vertex $H y^{n} x=H x y^{n}=H y^{n}$, hence this edge is a loop. The geodesic core of Cayley $(G, H)$ consists of a single vertex $H \cdot 1$ and a single edge $(H \cdot 1, x)$, which begins and ends at $H \cdot 1$. The diameter of the geodesic core of Cayley $(G, H)$ is 0 , because it has only one vertex, verifying again that $H$ is 0 -quasiconvex in $G$.

On the other hand, consider a subgroup $L=\langle x y\rangle$ of $G$. A path $p$ beginning at vertex 1 of Cayley $(G)$ with $\operatorname{Lab}(p) \equiv x^{n} y^{n}$ is a geodesic in Cayley $(G)$, and the vertex $x^{n}$ of Cayley $(G)$ is in $p$, but $d\left(x^{n}, L\right)=n$, so $L$ is not quasiconvex in Cayley $(G)$. In order to describe Cayley $(G, L)$, note that $\left\{L y^{n}, n \in Z\right\}$ is the set of all cosets of $L$ in $G$, so we consider it as the set of vertices of Cayley $(G, L)$. The edge $\left(L y^{n}, y\right)$ begins at the vertex $L y^{n}$ and ends at the vertex $L y^{n} y=L y^{n+1}$. However, the edge $\left(L y^{n}, x\right)$ begins at the vertex $L y^{n}$ and ends at the vertex $L y^{n} x=L x y y^{n-1}=L y^{n-1}$. The geodesic core of Cayley $(G, L)$ is the whole graph Cayley $(G, L)$ which is unbounded, demonstrating again that $L$ is not quasiconvex in $G$.

Recall that a path $p$ is an $L$-local geodesic if each subpath of $p$ of length at most $L$ is a geodesic. For example, any path in Cayley $(G)$ is a 1-local geodesic. A geodesic triangle in Cayley $(G)$ is a closed path $p=p_{1} p_{2} p_{3}$, where each $p_{i}$ is a geodesic. A group $G$ is $\delta$-negatively curved if each side of each geodesic triangle in Cayley $(G)$ belongs to the $\delta$-neighbourhood of the union of two other sides. We consider only negatively curved groups that are finitely generated.

We use the following well-known fact.

LEMMA 3 ([Gro]). Let $G$ be a $\delta$-negatively curved group, and let $L>4 \delta$. Then any L-local geodesic $p$ in Cayley $(G)$ belongs to the $M$-neighbourhood of a geodesic $\gamma$ joining the endpoints of $p$, where $M$ depends only on $L$ and on $\delta$.

\section{Quasiconvex subgroups}

Up to this point we worked with some fixed presentation $\langle X \mid R\rangle$ of $G$. Now we need to redefine the presentation. 
DEFINITION 5. Let $\langle X \mid R\rangle$ be a presentation of $G$, and let $2 L$ be a constant which is bigger than the length of the longest relator in $R$. Let $R^{\prime}$ be the set of all reduced words $w$ in $W(X)$ which represent $1_{G}$ such that $|w| \leq 2 L$. Then $\left\langle X \mid R^{\prime}\right\rangle$ is a presentation for $G$, and we use it for the rest of the paper.

DEFINITION 6. An $L$-local geodesic in Cayley $(G, H)$ is the image of an $L$-local geodesic in Cayley $(G)$ under the projection $\rho_{H}$.

LEMMA 4. Let $\left\langle X \mid R^{\prime}\right\rangle$ be the presentation of a group $G$, as in Definition 5 , let $C$ be a finite subcomplex of Cayley ${ }_{2}(G, H)$, and let $v_{0}$ be a vertex in $\operatorname{Cayley}_{2}(G, H) \backslash C$ with $d\left(v_{0}, C\right)<L$. Then any closed path $q$ in Cayley $(G, H) \backslash C$ beginning at $v_{0}$ is homotopic in Cayley $_{2}(G, H) \backslash C$ to a closed path $p$ in Cayley $(G, H) \backslash C$ beginning at $v_{0}$ with the following property: there exists a decomposition $p=p_{1} \cdots p_{n}$ such that $p_{2 i-1}$ is a maximal subpath of $p$ which belongs to the L-neighbourhood of $C$, and $p_{2 i}$ is an L-local geodesic in Cayley $(G, H) \backslash C$ with both endpoints distance L away from $C$.

PROOF. Let $t$ be a maximal subpath of $q$ which lies outside the $L$-neighbourhood of $C$ and is not an $L$-local geodesic in Cayley $(G, H)$. Then there exists a subpath $t^{\prime}$ of $t$ shorter than $L$, which is not a geodesic, so $t$ can be shortened by replacing $t^{\prime}$ with a shorter path $t^{\prime \prime}$. As $\bar{t}^{\prime} t^{\prime \prime}$ is a closed path which is shorter than $2 L$, the word $\operatorname{Lab}\left(\bar{t}^{\prime} t^{\prime \prime}\right)$ is a relator in $R^{\prime}$, so it bounds a 2-cell in $\operatorname{Cayley}_{2}(G, H)$. As $\left|t^{\prime \prime}\right|<L$, this cell lies in the complement of $C$ in Cayley $_{2}(G, H)$. As the path obtained from $q$ by replacing $t^{\prime}$ with $t^{\prime \prime}$ is homotopic to $q$ in Cayley $_{2}(G, H) \backslash C$, lies in the complement of $C$ in Cayley $(G, H)$ and is shorter than $q$, we obtain the required path $p$ after finitely many repetitions of above procedure.

LEMMA 5. Let $H$ be a $K$-quasiconvex subgroup of a $\delta$-negatively curved group $G$, let $L>4 \delta$, let $M$ be as in Lemma 3, and let $p$ be an L-local geodesic in Cayley $(G)$ with both endpoints distance $d$ away from $H$. Then $p$ belongs to the $(K+M+d+2 \delta)$ neighbourhood of $H$ in Cayley $(G)$.

PROOF. Let $s_{1}$ and $s_{2}$ be geodesics in Cayley $(G)$ of length at most $d$ joining a vertex $h_{1} \in H$ to the initial vertex of $p$, and the terminal vertex of $p$ to a vertex $h_{2} \in H$, respectively. Let $\gamma^{\prime}$ be a geodesic with the same endpoints, as $p$, and let $\gamma$ be a geodesic joining $h_{1}$ to $h_{2}$. As $H$ is $K$-quasiconvex in $G, \gamma$ belongs to the $K$-neighbourhood of $H$. Consider a closed path $s_{1} \gamma^{\prime} s_{2} \bar{\gamma}$ in Cayley $(G)$. This path is a geodesic 4-gon in a $\delta$-negatively curved group $G$, hence $\gamma^{\prime}$ belongs to the $2 \delta$-neighbourhood of the union of the other 3 sides. As $s_{1}$ and $s_{2}$ are shorter than $d$, it follows that $\gamma^{\prime}$ belongs to the $(K+d+2 \delta)$-neighbourhood of $H$. As $p$ is an $L$-local geodesic, Lemma 3 implies that it belongs to the $M$-neighbourhood of $\gamma^{\prime}$, proving Lemma 5. 
THEOREM 2. A quasiconvex subgroup $H$ of a negatively curved group $G$ is tame in $G$.

ProOF. Let $H$ be a $K$-quasiconvex subgroup of a $\delta$-negatively curved group $G$, and let $C$ and $v_{0}$ be as in Lemma 4 . Let $q$ be any closed path in Cayley $(G, H) \backslash C$ beginning at $v_{0}$. Let $p$ be a path equivalent to $q$, as described in Lemma 4. As $C$ is a finite graph, it belongs to the $\epsilon$-neighbourhood of $H \cdot 1$ in Cayley $(G, H)$ for some constant $\epsilon$. Then by construction, the subpaths $p_{2 i-1}$ of $p$ belong to the $(\epsilon+L)$-neighbourhood of $H \cdot 1$, hence the endpoints of the subpaths $p_{2 i}$ belong to the $(\epsilon+L)$-neighbourhood of $H \cdot 1$. Then Lemma 5 implies that the subpaths $p_{2 i}$ belong to the $(\epsilon+L+M+K+2 \delta)$-neighbourhood of $H \cdot 1$. Therefore, $q$ is homotopic in Cayley $_{2}(G, H) \backslash C$ to a path in the $(\epsilon+L+M+K+2 \delta)$-neighbourhood of $H \cdot 1$. As $G$ is finitely generated, this neighbourhood is a finite graph, proving Theorem 2 .

REMARK. Recall that a Dehn presentation for the group $G$ is a finite presentation $\langle X \mid R\rangle$ such that any non-trivial reduced word $w \in W(X)$ representing $1_{G}$ contains more than half of some $r \in R$, that is, there exists a decomposition $r \equiv r_{1} r_{2}$ with $\left|r_{1}\right|>\left|r_{2}\right|$ such that $w \equiv w_{1} r_{1} w_{2}$. A group with a Dehn presentation has the following algorithm, known as the Dehn algorithm, for a solution of the word problem. Let $L$ be the length of the longest relator in $R$. Given a word $w \in W(X)$, check all its subwords which are shorter than $L+1$. If none of these subwords is a bigger half of some relator in $R$, then $w$ does not represent $1_{G}$. Otherwise, there exists a relator $r=r_{1} r_{2} \in R$ with $\left|r_{1}\right|>\left|r_{2}\right|$ such that $w$ contains $r_{1}$ as a subword. But then the word $w^{\prime}$ obtained from $w$ by replacing $r_{1}$ by $r_{2}^{-1}$ is equivalent to $w$ in $G$ and is shorter than $w$, so the algorithm terminates after finitely many steps. We would like to point out that the proof of Lemma 4 is based on a similar procedure.

\section{Acknowledgement}

The author thanks Gopal Prasad, Frank Raymond, John Stallings and Gadde Swarup for their support.

\section{References}

[Bon] F. Bonahon, 'Bouts ses variétés hyperboliques de dimension 3', Ann. of Math. 124 (1986), 71-158.

[Gab] D. Gabai, 'Problems in foliations and laminations', in: AMS/IP Studies in Advanced Mathematics vol. 2 part 2 (ed. W. Kazez) (AMS and International Press, Providence, 1997) pp. 1-35.

[Git] R. Gitik, 'On quasiconvex subgroups of negatively curved groups', J. Pure Appl. Algebra 119 (1997), 155-169. 
[Gro] M. Gromov, 'Hyperbolic groups', in: Essays in group theory (ed. S. M. Gersten) MSRI publications vol. 8 (Springer, New York, 1987) pp. 75-263.

[Kir] R. Kirby, 'Problems in low-dimensional topology', in: AMS/IP Studies in Advanced Mathematics vol. 2 part 2 (ed. W. Kazez) (AMS and International Press, Providence, 1997) pp. 35-473.

[Mil] M. Mihalik, 'Compactifying coverings of 3-manifolds', Comment. Math. Helv. 71 (1996), 362372.

[Mi2] — 'Group extensions and tame pairs', Trans. Amer. Math. Soc. 351 (1999), 1095-1107.

[Poé] V. Poénaru, 'Almost convex groups, Lipschitz combing, and $\pi_{1}^{\infty}$ for universal covering spaces of closed 3-manifolds', J. Differential Geom. 35 (1992), 103-130.

[Sim] J. Simon, 'Compactification of covering spaces of compact 3-manifolds', Michigan Math. J. 23 (1976), 245-256.

[Thu] W. Thurston, Geometry and topology of 3-manifolds (Princeton University, Princeton, 1978).

[Tuc] T. W. Tucker, 'Non-compact 3-manifolds and the missing boundary problem', Topology 13 (1974), 267-273.

A \& $\mathrm{H}$ Consultants

Ann Arbor, MI 48104

USA

e-mail: ritagtk@math.lsa.umich.edu 